\title{
Thermoelectric Signature of Individual Skyrmions
}

\author{
Alexander Fernández Scarioni $\odot,{ }^{1}$ Craig Barton $\odot,{ }^{2}$ Héctor Corte-León $\odot,{ }^{2}$ Sibylle Sievers $\odot,{ }^{1}$ \\ Xiukun Hu๑, ${ }^{1}$ Fernando Ajejas $\odot,{ }^{3}$ William Legrand $\odot,{ }^{3}$ Nicolas Reyren $\odot,{ }^{3}$ Vincent Cros, ${ }^{3}$ \\ Olga Kazakova, ${ }^{2}$ and Hans W. Schumacher ${ }^{1}$ \\ ${ }^{1}$ Physikalisch-Technische Bundesanstalt, 38116 Braunschweig, Germany \\ ${ }^{2}$ National Physical Laboratory, Teddington TW110LW, United Kingdom \\ ${ }^{3}$ Unité Mixte de Physique, CNRS, Thales, Université Paris-Saclay, 91767 Palaiseau, France
}

(Received 7 April 2020; revised 26 June 2020; accepted 2 November 2020; published 16 February 2021)

\begin{abstract}
We experimentally study the thermoelectrical signature of individual skyrmions in chiral $\mathrm{Pt} / \mathrm{Co} / \mathrm{Ru}$ multilayers. Using a combination of controlled nucleation, single skyrmion annihilation, and magnetic field dependent measurements the thermoelectric signature of individual skyrmions is characterized. The observed signature is explained by the anomalous Nernst effect of the skyrmion's spin structure. Possible topological contributions to the observed thermoelectrical signature are discussed. Such thermoelectrical characterization allows for noninvasive detection and counting of skyrmions and enables fundamental studies of topological thermoelectric effects on the nanoscale.
\end{abstract}

DOI: 10.1103/PhysRevLett.126.077202

Skyrmions are nanoscale topologically nontrivial spin structures which are inherently robust due to their particular topology and can be driven efficiently by electrical currents [1-3], with potential applications in logic and storage devices and neuromorphic computing [4]. Their electrical characterization and manipulation have been investigated intensely over recent years [5-14] leading to observations such as the skyrmion Hall effect $[15,16]$ and the topological Hall effect $[14,17]$. However, only very few studies have addressed or exploited their thermoelectrical properties [18-21]. While these studies mainly address the topological properties of bulk materials, in thin films the thermoelectrical signal has recently been used as a tool to image skyrmions [21]. In conventional spintronic materials thermoelectric studies have led to important discoveries such as the tunneling magneto-Seebeck effect [22] or spin heat accumulation [23] and have enabled nanoscale detection of domain wall motion [24]. For skyrmions, such thermoelectric studies could shine light on topological contributions to the Nernst effect [25] and might provide new tools for skyrmion detection and manipulation.

In this Letter, we experimentally study the thermoelectrical signature of individual skyrmions in a $\mathrm{Pt} / \mathrm{Co} / \mathrm{Ru}$ multilayer microstripe and attribute it unambiguously to the anomalous Nernst effect (ANE) originating from the spin structure of the skyrmion without significant topological

Published by the American Physical Society under the terms of the Creative Commons Attribution 4.0 International license. Further distribution of this work must maintain attribution to the author(s) and the published article's title, journal citation, and DOI. contributions. Our experiments show that beyond this fundamental insight into the thermoelectrical properties of individual nanoscale topological spin structures our findings enable noninvasive characterization, detection and counting of skyrmions in magnetic microdevices.

We use micron-wide stripes made of $\mathrm{Pt} / \mathrm{Co} / \mathrm{Ru}$ multilayers with perpendicular magnetic anisotropy (PMA) and strong interfacial Dzyaloshinskii-Moriya interaction (DMI) as shown in Fig. 1(a). The multilayer stack is comprised of asymmetric trilayers based on $\mathrm{Ta}(5) / \mathrm{Pt}(8) /[\mathrm{Co}(1) /$ $\mathrm{Ru}(1.4) / \mathrm{Pt}(0.6)] \times 10 / \mathrm{Pt}(2.4)$ (numbers are thickness in $\mathrm{nm}$ ) deposited on a thermally oxidized Si substrate (methods in Ref. [26]). The microstripes were patterned by electron beam lithography and argon etching. Sputter deposited contacts and a $200 \mathrm{~nm}$ wide microheater of $5 \mathrm{~nm} \mathrm{Ta}$ and $95 \mathrm{~nm}$ Pt were patterned by lift-off. The microheater is used to induce a transverse in-plane thermal gradient $\nabla T_{x}$ in the microstripe. $\nabla T_{x}$ is perpendicular to the PMA magnetization $\mathbf{M}$ resulting in an ANE voltage $V_{\mathrm{ANE}}$ in the longitudinal direction between the two contacts following

$$
V_{\mathrm{ANE}}=-N_{\mathrm{ANE}} \mu_{0} l \overline{\nabla T_{x}} M_{z}
$$

Here $N_{\mathrm{ANE}}$ is the ANE coefficient per magnetic moment, $\mu_{0}$ is the vacuum permeability, $l$ is the length of the sensed region, $\overline{\nabla T_{x}}$ is the averaged $x$ component of the thermal gradient across the material, and $M_{z}$ is the averaged $z$ component of the magnetization in the sensing region between the measurement contacts [Fig. 1(a)]. In our study the saturation condition is denoted by $M_{z}=M_{s}$, the saturation magnetization. 


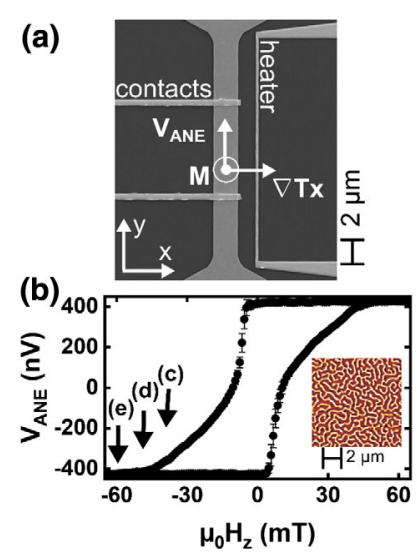

(c)
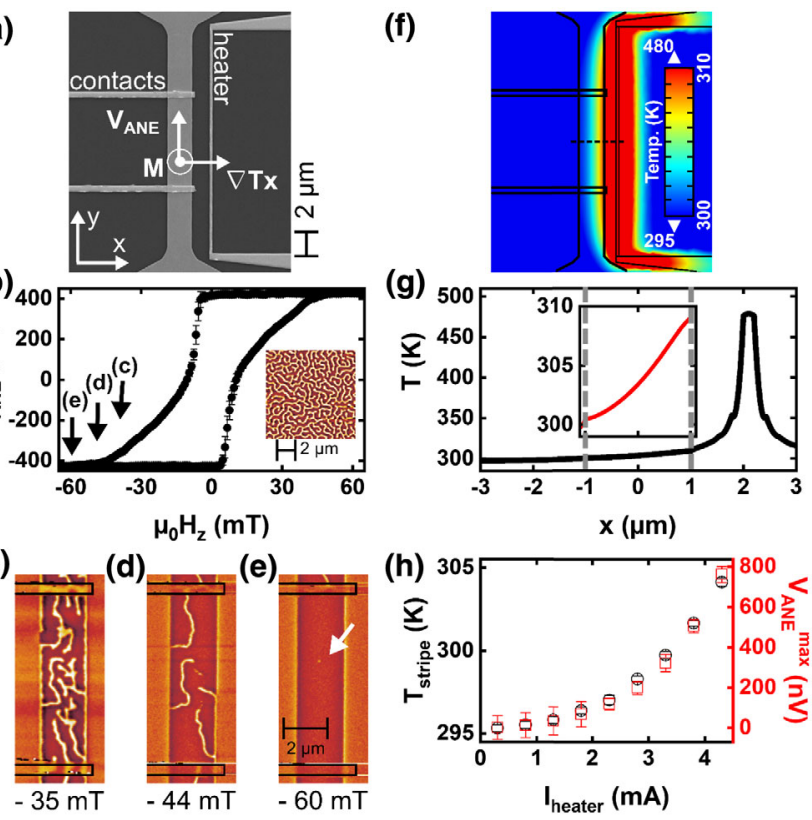

FIG. 1. (a) Scanning electron micrograph of the device. (b) ANE voltage as a function of the applied out-of-plane field. The black arrows mark the corresponding domain states imaged by MFM shown in (c)-(e). The inset shows an MFM image of the film before patterning in zero field. (c)-(e) MFM images of the microstripe for increasingly negative values of the total out-ofplane magnetic field. Here, the stated field is the sum of the applied field and the tip stray field at the sample surface. The white arrow indicates a skyrmion. (f) Simulated temperature distribution for $I_{\text {heater }}=4.3 \mathrm{~mA}$. (g) Simulated temperature profile along the dashed line in (f). (h) Measured temperature rise (black circles) as a function of applied current and ANE amplitude (red squares) in the microstripe vs. $I_{\text {heater }}$.

The ANE in the device was first characterized by magnetization reversal hysteresis loops. The hysteresis loops as well as all the subsequent ANE voltage measurements were performed at ambient conditions. In the ANE voltage measurements, $V_{\mathrm{ANE}}$ was detected at the second harmonic using a lock-in technique [27]. Figure 1(b) shows a hysteresis loop of $V_{\mathrm{ANE}}$ as function of the perpendicularly applied out-of-plane field $\mu_{0} H_{z}$. The $z$ component of the averaged magnetization, $M_{z}$, shows the expected reversal behavior of PMA samples with maze domains as observed in the nonpatterned thin film by magnetic force microscopy (MFM) at zero applied magnetic field [inset of Fig. 1(b)]. With increasing applied field, regions of reversed magnetization expand until full saturation is reached as can be seen in MFM micrographs of the microstripe in different applied fields [Figs. 1(c)-1(e)]. Note that in saturation the thermoelectric voltage can only be due to the ANE in our measuring geometry which is shunted by the $\mathrm{Pt}$ in the multilayer (see Ref. [27]). Additionally, individual skyrmions [white arrow in Fig. 1(e)] occur, demonstrating field stabilization of skyrmions during the reversal loops.
To determine the temperature distribution and $\overline{\nabla T_{x}}$, finite element heat flux modeling of the device was performed [27]. The resulting temperature distribution for a dc microheater current $I_{\text {heater }}$ of $4.3 \mathrm{~mA}$ is shown in Fig. 1(f). The average thermal gradients along the $x, y, z$ axes in the device are found to be $\overline{\nabla T_{x}}=4.2 \mathrm{~K} / \mu \mathrm{m}, \overline{\nabla T_{y}}=$ $0.2 \mathrm{mK} / \mu \mathrm{m}$, and $\quad \overline{\nabla T_{z}}=2.9 \mathrm{mK} / \mu \mathrm{m}$, respectively. Given the order of magnitude difference between $\overline{\nabla T_{x}}$, and $\overline{\nabla T_{y}}$ as well as $\overline{\nabla T_{z}}$, we neglect the latter two contributions to the ANE in further analysis (see Ref. [27]). In Fig. 1(g), we show the simulated surface temperature profile across the microstripe along the dashed line in Fig. 1(f). Inside the microstripe (inset), the temperature $T_{\text {stripe }}$ increases by about $8.6 \mathrm{~K}$. Using the microstripe as a resistive thermometer, the averaged $T_{\text {stripe }}$ can also be experimentally determined as function of $I_{\text {heater }}$ [27]. As shown in Fig. 1(h) (black circles), $T_{\text {stripe }}$ increases quadratically with $I_{\text {heater }}$ as expected for Joule heating and the experimentally determined temperature increase of $8.6 \mathrm{~K}$ for the maximum $I_{\text {heater }}=4.3 \mathrm{~mA}$ agrees well with the simulations.

Similar ANE hysteresis loops as in Fig. 1(b) were measured for a range of heater currents $I_{\text {heater }}=$ (0.2-4.3) mA. In Fig. 1(h), we show the ANE amplitude $V_{\mathrm{ANE}}^{\max }$ (red squares) derived from subtracting the measured saturated $V_{\mathrm{ANE}}^{\mathrm{sat}}$ for positive and negative fields. Again, $V_{\mathrm{ANE}}^{\max }$ scales quadratically with $I_{\text {heater }}$ and thus proportionally with $\overline{\nabla T_{x}}$ confirming the thermoelectrical origin. Using Eq. (1), the measured $V_{\mathrm{ANE}}^{\max }$ with the average $\overline{\nabla T_{x}}$ from the temperature calibration or simulations and $\mu_{0} M_{s}=$ $1.4 \mathrm{~T}$ from magnetometry data, a Nernst coefficient of $N_{\mathrm{ANE}}=8.1 \mathrm{nV} / \mathrm{KT}$ is derived.

To controllably study the thermoelectrical response of few or individual skyrmions, we use a combination of current induced nucleation [8-13] and magnetic fields to generate skyrmions in the microstripe, and image them by in situ MFM.

MFM images of the microstripe before and after driving a single rectangular current pulse with nominal duration of $200 \mathrm{~ns}$ through the microstripe are displayed in Figs. 2(a)-2(d). Applied pulse current densities $j$ ranged from $3.34 \times 10^{11}$ to $3.97 \times 10^{11} \mathrm{~A} / \mathrm{m}^{2}$ and the applied magnetic fields $\mu_{0} H_{z}=(0.49-40.7) \mathrm{mT}$. Before the pulse, an initial maze domain state was obtained by first saturating the sample at negative field and then sweeping to the desired positive field value. Depending on the relative intensities of $j$ and $\mu_{0} H_{z}$, various end states were found that could be classified into the following three categories: pure stripe domains [Fig. 2(a)], coexistence of skyrmions and stripe domains [Fig. 2(b)], or pure skyrmions [Figs. 2(c) and 2(d)], as summarized in the nucleation phase diagram, Fig. 2(e). The dotted line marks the region where single digit numbers of skyrmions are nucleated (blue triangles). Repeated measurements did not reveal preferential 

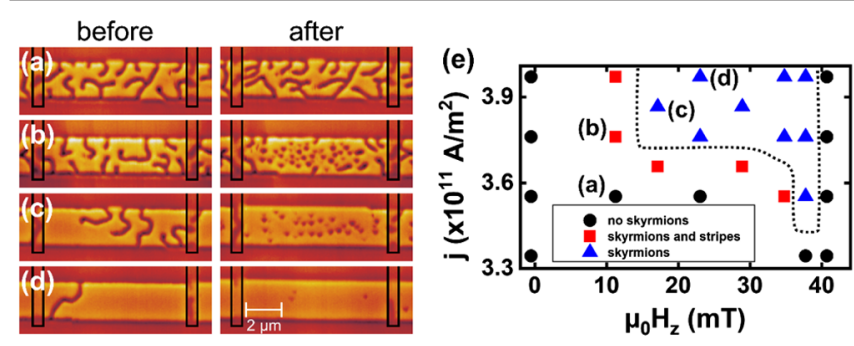

FIG. 2. (a)-(d) MFM measurements of the microstripe before and after application of single 200-ns-long current pulses. (a) Current density $j=3.55 \times 10^{11} \mathrm{~A} / \mathrm{m}^{2}$, magnetic field $\mu_{0} H_{z}=11.28 \mathrm{mT}$. (b) $j=3.76 \times 10^{11} \mathrm{~A} / \mathrm{m}^{2}, \mu_{0} H_{z}=11.28 \mathrm{mT}$. (c) $j=3.86 \times 10^{11} \mathrm{~A} / \mathrm{m}^{2}, \quad \mu_{0} H_{z}=17.16 \mathrm{mT}$. (d) $j=3.97 \times$ $10^{11} \mathrm{~A} / \mathrm{m}^{2}, \mu_{0} H_{z}=23.05 \mathrm{mT}$. (e) Nucleation phase diagram for skyrmion generation. The parameter range suitable for skyrmion nucleation is indicated by the dotted black line.

nucleation sites but rather stochastic skyrmion distributions indicating a thermally driven nucleation process [13].

Although the above protocol allows us to generate single digit numbers of skyrmions it does not allow us to controllably change the number of skyrmions. Therefore, we applied a single skyrmion annihilation procedure exploiting the confined stray magnetic field around the MFM tip apex [42,43]. In Fig. 3(a), we show an MFM image after nucleation of five skyrmions in an applied magnetic field of $+34.84 \mathrm{mT}$. For an external applied magnetic field below $+24 \mathrm{mT}$, the local total field (the sum of the external applied field and the local tip stray field) under the MFM tip is below the skyrmion annihilation field (between 50 and $60 \mathrm{mT}$ ). This allows us to image the
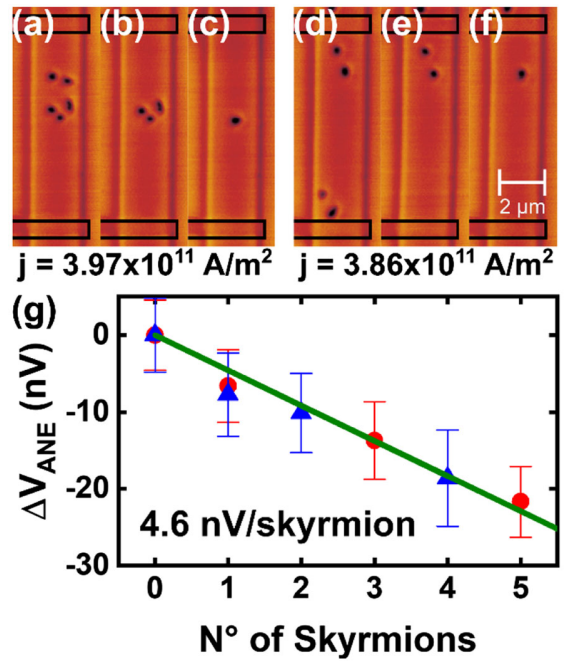

FIG. 3. Two independent sequences of MFM images, (a)-(c) and (d)-(f), demonstrating the probe induced annihilation process of individual skyrmions. (g) $\Delta V_{\mathrm{ANE}}$ as function of the number of skyrmions in the sensing area corresponding to the skyrmion configurations (a)-(f). The red dots correspond to (a)-(c); the blue triangles correspond to (d)-(f). The green line is a linear fit, yielding an average Nernst voltage of $4.6 \pm 0.2 \mathrm{nV}$ per skyrmion. skyrmions without detrimental tip-sample interaction. In contrast, when the external field exceeds $+24 \mathrm{mT}$, the total local field under the MFM tip exceeds the annihilation threshold. The targeted application of external fields thus allows us to controllably delete individual nanoscale skyrmions by placing the MFM tip above them. Two examples of this selective annihilation process are shown in the MFM micrograph sequences, Figs. 3(a)-3(c) and Figs. 3(d)-3(f). Here, the external field was set to $+28 \mathrm{mT}$ when scanning over the skyrmion that was to be erased, which, in combination with the MFM tip's field was sufficient to annihilate skyrmions. Note that no skyrmion motion was observed whilst scanning the MFM tip over them, indicating substantial pinning.

Importantly, our experimental setup allows in situ MFM characterization and annihilation of the skyrmions simultaneously with the thermoelectrical characterization. This allows, for the first time, the measurement of the thermoelectrical signature of individual skyrmions, analogously to previous studies of the thermoelectrical signature of an individual magnetic domain wall in a nanowire [24] or of the anomalous Hall effect detection of skyrmions [11]. The measured ANE signal from the skyrmion configurations shown in Figs. 3(a)-3(c) and Figs. 3(d)-3(f) is plotted in Fig. $3(\mathrm{~g})$, as red dots and blue triangles, respectively. The difference $\Delta V_{\mathrm{ANE}}$ of the measured ANE signal to the ANE in saturation $\left(\Delta V_{\mathrm{ANE}}=V_{\mathrm{ANE}}-V_{\mathrm{ANE}}^{\mathrm{sat}}\right)$ is plotted as a function of the number of detected skyrmions in the sensing region. The data point for zero skyrmions corresponds to the saturated signal $V_{\mathrm{ANE}}^{\text {sat }}$ where, by definition, $\Delta V_{\mathrm{ANE}}=0$. The error bars reflect the standard deviation of the measurement noise. $\Delta V_{\mathrm{ANE}}$ shows a clear linear dependence on the skyrmion number. By linear regression with fixed zero intercept an average ANE voltage signature per skyrmion of $V_{\mathrm{ANE}}^{\mathrm{sky}}=4.6 \pm 0.2 \mathrm{nV}$ is derived for the given experimental conditions, i.e., $\mu_{0} H_{z}=+11.3 \mathrm{mT}$, $\overline{\nabla T_{x}}=4.2 \mathrm{~K} / \mu \mathrm{m}$.

Following Eq. (1), the measured $\Delta V_{\mathrm{ANE}}$ is proportional to the net $M_{z}$ in the area between the two measurement contacts, and hence proportional to the cumulative skyrmion induced change of the magnetization. The observed reduction of $\Delta V_{\mathrm{ANE}}$ with the number of skyrmions should thus, in a strict sense, scale with the net reversed magnetization area inside the skyrmions rather than with the skyrmion number. We estimate an effective skyrmion area $A_{\text {sky }}$ of each individual skyrmion from the MFM measurements [27]. The lower left inset to Fig. 4(a) shows a zoomin view of an MFM image of a typical skyrmion with the derived skyrmion area $A_{\text {sky }}$ marked by the white line. The upper right inset demonstrates a very good agreement of a profile through one skyrmion extracted from an experimental MFM phase shift signal (black) with a Gaussian fit (red). The main panel in Fig. 4(a) displays $\Delta V_{\mathrm{ANE}}$ all data collected from three nucleation and erasure sequences (the red dots, blue triangles, and black squares correspond to 

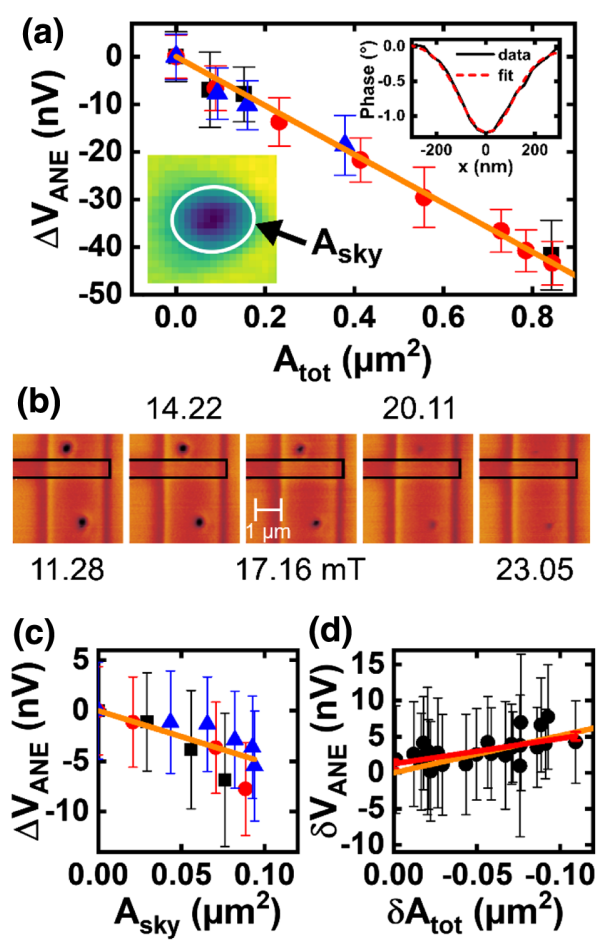

FIG. 4. (a) ANE signal as a function of the total effective reversed area. The different symbols correspond to three nucleation/annihilation sequences [same in (c)]. The orange line represents the calculated ANE voltage for the given reversed magnetization area [same in (c) and (d)]. Left inset: MFM image of a single skyrmion with the derived $A_{\text {sky }}$. Right inset: section through the MFM phase signal (black) of the same skyrmion and Gaussian-fit data (red). (b) MFM images showing the field dependence of a single skyrmion. (c) ANE voltage as a function of total skyrmion area $A_{\text {sky }}$ at different magnetic fields. (d) Differential ANE voltages $\delta V_{\text {ANE }}$ plotted against the difference in reversed area. The red line is a linear fit of the data allowing for a nonzero intercept.

separate measurement sequences [27]) including the data from figure Fig. 3(g) (same plot marks) as function of the total reversed skyrmion area when $n$ skyrmions are present, $A_{\text {tot }, n}=\sum_{i=1}^{n} A_{\text {sky }, i}$ summed over all $n$ skyrmions inside the sensing area. Again, a linear behavior is observed. The orange line is the calculated ANE signal considering the average thermal gradient $\overline{\nabla T_{x}}$, the estimated $N_{\mathrm{ANE}}$ and the total reversed skyrmion area $A_{\text {tot }, n}$. Apparently, the measured voltage can be very well described by the ANE contribution of the reversed skyrmion area, strongly supporting our assumption, that the ANE is the dominant contribution to the measured thermoelectric voltage [27]. Note that the thermal simulations of Fig. 1(f) suggest a spatial variation of approximately $50 \%$ in comparison to the average $\overline{\nabla T_{x}}$ over the wire width which would result in a dependence of the skyrmion ANE on their $x$ position which, however, is not observed experimentally. This indicates that the spatial variation of $\nabla T_{x}$ is overestimated by the simulations [27].
Additionally, we investigate the change of the ANE response of a single skyrmion as a function of the skyrmion area as tuned by an out-of-plane magnetic field. Figure 4(b) shows a sequence of five MFM micrographs of a single skyrmion in different fields. The variation $\Delta V_{\mathrm{ANE}}$ as a function of the skyrmion area of Fig. 4(b) is plotted in Fig. 4(c) as blue triangles. The red dots and black squares correspond to two additional separate measurement sequences on different skyrmions [27]. Again, the ANE signal calculated from the skyrmion area (orange line) describes well the single skyrmion data within the experimental measurement uncertainty.

It has been observed that when spin polarized electrons transverse a topologically nontrivial spin texture, such as a magnetic skyrmion, they can accumulate a Berry phase [44] in analogy to an Aharonov-Bohm phase resulting from a fictious magnetic field antiparallel to the uniform magnetization [45]. Experimentally this accumulated Berry phase can result in a topological Hall effect [17] or a topological Nernst effect [25] as observed in the skyrmion phase of bulk MnGe [18] and MnSi [19] as well as in $\mathrm{Mn}_{1.8} \mathrm{PtSn}$ thin films [20].

The topological contribution $V_{\mathrm{ANE}}^{\text {top }}$ to the measured Nernst signal $V_{\text {ANE }}$ should scale with the total topological charge of the skyrmions inside the microwire. Our micromagnetic simulations show, that for all skyrmion sizes occurring in the experiment, the skyrmions should exhibit the same topological charge $\xi_{\text {top }}$ as shown in the Supplemental Material [27]. Furthermore, the chirality or the helicity, which is the sense of rotation of the spins relative to each other, do not influence the topological charge [46]. Therefore, the topological contribution should scale with the total number of skyrmions, $n$, like $V_{\mathrm{ANE}}^{\text {top }}=$ $n \xi_{\text {top }}$ and not with their net area $A_{\text {tot }, n}$. Thus, in the presence of a significant $V_{\mathrm{ANE}}^{\text {top }}$ the total measured thermovoltage signal $V_{\text {tot }, n}=V_{\text {ANE }}\left(A_{\text {tot }, n}\right)+n \xi_{\text {top }}$, should not be proportional to $A_{\mathrm{tot}, n}$, However, within the experimental uncertainties such nonlinearity indicating a significant $V_{\mathrm{ANE}}^{\mathrm{top}}$ is not visible in Figs. 4(a) and 4(c).

For a more in-depth analysis, we extract the incremental $\Delta V_{\mathrm{ANE}}$ increases $\delta V_{\mathrm{ANE}, n}=\Delta V_{\mathrm{ANE}, n}-\Delta V_{\mathrm{ANE}, n-1}$ being the change of $\Delta V_{\mathrm{ANE}, i}$ when annihilating skyrmion $n$ and thus changing the number of skyrmions (and thus the topological charge of the system) by one. It is plotted against the corresponding incremental area change $\delta A_{\mathrm{tot}, n}=-\left(A_{\mathrm{tot}, n}-A_{\mathrm{tot}, n-1}\right)$. This allows to separate $V_{\mathrm{ANE}}$ and $V_{\mathrm{ANE}}^{\mathrm{top}}$ contributions since

$$
\delta V_{\mathrm{ANE}, n}=-N_{\mathrm{ANE}} \mu_{0} \overline{\nabla T_{x}} 2 M_{s} \delta A_{\mathrm{tot}, n} / w+1 \cdot \xi_{\mathrm{top}},
$$

and thus $\xi_{\text {top }}$ can be extracted as an offset. The width of the microstripe is represented by $w$. Figure 4(d) shows $\delta V_{\mathrm{ANE}, n}\left(\delta A_{\mathrm{tot}, n}\right)$ for all datasets in the study. Again, the predicted ANE signal (orange line) well describes the data 
within the experimental uncertainty. However, by allowing a free linear fit to the data, we obtain a nonzero intercept at zero skyrmion area indicating a potential topological contribution $V_{\mathrm{ANE}}^{\mathrm{top}}$ to the skyrmion Nernst signature independent of $A_{\text {sky }}$. However, we emphasize that the derived interception of $1.2 \pm 0.6 \mathrm{nV}$ is about four times smaller than the ANE contribution per skyrmion $(4.6 \pm 0.2 \mathrm{nV})$, but numerically different from zero within $2 \sigma$ confidence. Based on this dataset, a topological contribution thus cannot be ruled out. Note that such a contribution has been found negligible in pure electrical detection in similar skyrmion multilayers [11]. Considering the statistical uncertainty of our data, further studies are required to unambiguously conclude on the nature of the topological Nernst signature of individual skyrmions. One explanation to the rather low contribution may arise from the fact that for the metallic multilayers studied here, both the electron's mean free path and the spin diffusion length are of the order of few nanometers $[14,47]$ and thus significantly smaller than the average skyrmion diameter. The resulting strong scattering might provide a hypothesis for the absence of a strong topological Nernst contribution in our experimental data. Reducing the skyrmion radius and increasing the density of the skyrmions might be beneficial to experimentally observe the topological Nernst effect in metallic multilayer samples.

In conclusion, we have measured and characterized the thermoelectrical signature of individual nanoscale skyrmions in $\mathrm{Pt} / \mathrm{Co} / \mathrm{Ru}$ multilayers enabling noninvasive all-electrical detection and counting of skyrmions in magnetic devices. The thermoelectric signature is well explained by the ANE of the reversed magnetization area of the individual skyrmions. Only weak indications of an additional topological Nernst contribution are present in the data. Future experiments using material systems with smaller skyrmion diameters or larger mean free paths of the conduction electrons could allow to unequivocally identify the topological Nernst contribution of individual skyrmions.

This work was supported in part by the European Metrology Research Programme (EMRP) and EMRP participating countries under the European Metrology Programme for Innovation and Research (EMPIR) Project No. 17FUN08-TOPS Metrology for topological spin structures. The work was co-funded by the Deutsche Forschungsgemeinschaft under Germany's Excellence Strategy-EXC-2123 QuantumFrontiers-390837967. Financial support from the Agence Nationale de la Recherche, France, under Grant Agreement No. ANR-17CE24-0025 (TOPSKY), the Horizon2020 Framework Programme of the European Commission, the Defense Advanced Research Projects Agency (DARPA) Topological Excitations in Electronics (TEE) program, through Grant MIPR No. HR0011831554 under
FET-Proactive Grant Agreement No. 824123 (SKYTOP) is acknowledged. We acknowledge the support of the UK government department for Business, Energy, and Industrial Strategy (IS) through the UK national quantum technologies programme (Low Loss Electronics project).

Note added.-After the submission of our manuscript Wang et al. reported the observation of discrete jumps in the anomalous Nernst voltage in $\mathrm{Ta} / \mathrm{CoFeB} / \mathrm{MgO}$ multilayers that they attributed to the field induced disappearance of single skyrmions [48].

[1] G. Finocchio, F. Büttner, R. Tomasello, M. Carpentieri, and M. Kläui, Magnetic skyrmions: From fundamental to applications, J. Phys. D 49, 423001 (2016).

[2] A. Fert, N. Reyren, and V. Cros, Magnetic skyrmions: Advances in physics and potential applications, Nat. Rev. Mater. 2, 17031 (2017).

[3] K. Everschor-Sitte, J. Masell, R. M. Reeve, and M. Kläui, Perpestive: Magnetic skyrmions- overview of recent progress in an active research field, J. Appl. Phys. 124, 240901 (2018).

[4] J. Zázvorka et al. Thermal skyrmion diffusion used in a reshuffler device, Nat. Nanotechnol. 14, 658 (2019).

[5] W. Jiang et al. Blowing magnetic skyrmion bubbles, Science 349, 283 (2015).

[6] S. Woo et al. Observation of room-temperature magnetic skyrmions and their current-driven dynamics in ultrathin metallic ferromagnets, Nat. Mater. 15, 501 (2016).

[7] C. Moreau-Luchaire et al. Additive interfacial chiral interaction in multilayers for stabilization of small individual skyrmions at room temperature, Nat. Nanotechnol. 11, 444 (2016).

[8] H. Hrabec, J. Sampaio, M. Belmeguenai, I. Gross, R. Weil, S. M. Chérif, A. Stashkevich, V. Jacques, A. Thiaville, and S. Rohart Current-induced skyrmion generation and dynamics in symmetric bilayers, Nat. Commun. 8, 15765 (2017).

[9] W. Legrand, D. Maccariello, N. Reyren, K. Garcia, C. Moutafis, C. Moreau-Luchaire, S. Collin, K. Bouzehouane, V. Cros, and A. Fert Room-Temperature Current-Induced Generation and Motion of sub-100 nm Skyrmions, Nano Lett. 17, 2703 (2017).

[10] F. Büttner et al. Field-free deterministic ultrafast creation of magnetic skyrmions by spin-orbit torques, Nat. Nanotechnol. 12, 1040 (2017).

[11] D. Maccariello, W. Legrand, N. Reyren, K. Garcia, K. Bouzehouane, S. Collin, V. Cros, and A. Fert Electrical detection of single magnetic skyrmions in metallic multilayers at room temperature, Nat. Nanotechnol. 13, 233 (2018).

[12] K. Zeissler et al. Discrete Hall resistivity contribution from Néel skyrmions in multilayer nanodiscs, Nat. Nanotechnol. 13, 1161 (2018).

[13] I. Lemesh et al. Current-induced skyrmion generation through morphological thermal transitions in chiral ferromagnetic heterostructures, Adv. Mater. 30, 1805461 (2018).

[14] M. Raju, A. Yagil, A. Soumyanarayanan, A. K. C. Tan, A. Almoalem, F. Ma, O. M. Auslaender, and C. Panagopoulos, 
The evolution of skyrmions in $\mathrm{Ir} / \mathrm{Fe} / \mathrm{Co} / \mathrm{Pt}$ multilayers and their topological Hall signature, Nat. Commun. 10, 696 (2019).

[15] W. Jiang et al. Direct observation of the skyrmion Hall effect, Nat. Phys. 13, 162 (2017).

[16] K. Litzius et al. Skyrmion Hall effect revealed by direct timeresolved x-ray microscopy, Nat. Phys. 13, 170 (2017).

[17] A. Neubauer, C. Pfleiderer, B. Binz, A. Rosch, R. Ritz, P. G. Niklowitz, and P. Boni, Topological Hall Effect in the A Phase of MnSi, Phys. Rev. Lett. 102, 186602 (2009).

[18] Y. Shiomi, N. Kanazawa, K. Shibata, Y. Onose, and Y. Tokura, Topological Nernst effect in a three-dimensional skyrmion-lattice phase, Phys. Rev. B 88, 064409 (2013).

[19] Y. Hirokane, Y. Tomioka, Y. Imai, A. Maeda, and Y. Onose, Longitudinal and transverse thermoelectric transport in MnSi, Phys. Rev. B 93, 014436 (2016).

[20] R. Schlitz, P. Swekis, A. Markou, H. Reichlova, M. Lammel, J. Gayles, A. Thomas, K. Nielsch, C. Felser, and S. T. B. Goennenwein All electrical access to topological transport features in Mn1.8PtSn films, Nano Lett. 19, 2366 (2019).

[21] R. Iguchi, S. Kasai, K. Koshikawa, N. Chinone, S. Suzuki, and K.-i. Uchida Thermoelectric microscopy of magnetic skyrmions, Sci. Rep. 9, 18443 (2019).

[22] T. Kuschel, M. Czerner, J. Walowski, A. Thomas, H. W. Schumacher, G. Reiss, C. Heiliger, and M. Münzenberg Tunnel magneto-Seebeck effect, J. Phys. D 52, 133001 (2019).

[23] F. K. Dejene, J. Flipse, G. E. Bauer, and B. J. Wees, Spin heat accumulation and spin-dependent temperatures in nanopillar spin valves, Nat. Phys. 9, 636 (2013).

[24] P. Krzysteczko et al. Nanoscale thermoelectrical detection of magnetic domain wall propagation, Phys. Rev. B 95, 220410(R) (2017).

[25] Y. P. Mizuta and F. Ishii, Large anomalous Nernst effect in a skyrmion crystal, Sci. Rep. 6, 28076 (2016).

[26] W. Legrand, D. Maccariello, F. Ajejas, S. Collin, A. Vecchiola, K. Bouzehouane, N. Reyren, V. Cros, and A. Fert Roomtemperature stabilization of antiferromagnetic skyrmions in synthetic antiferromagnets, Nat. Mater. 19, 34 (2020).

[27] See Supplemental Material at http://link.aps.org/ supplemental/10.1103/PhysRevLett.126.077202 for details on methods, discussion of spurious spin caloritronics contributions to the measurements, finite element modeling and the thermal gradient, device thermometry, estimation of the skyrmion area, skyrmion nucleation experiment, skyrmion field dependency and discussion of uncertainty analysis and simulated skyrmion magnetization distribution, which includes Refs. [28-41].

[28] E. Saitoh, M. Ueda, and H. Miyajima. Conversion of spin current into charge current at room temperature: Inverse spin-Hall effect, Appl. Phys. Lett. 88, 182509 (2006).

[29] P. Krzysteczko, X. Hu, N. Liebing, S. Sievers, and H. W. Schumacher, Domain wall magneto-Seebeck effect, Phys. Rev. B 92, 140405(R) (2015).

[30] A. Slachter, F. L. Bakker, and B. J. van Wees, Anomalous Nernst and anisotropic magnetoresistive heating in a lateral spin valve, Phys. Rev. B 84, 020412(R) (2011).

[31] COMSOL Multiphysics® v. 5.0, www.comsol.com, COMSOLAB, Stockholm, Sweden.
[32] A. S. Okhotin, A. S. Pushkarskii, and V. V. Gorbacher, Thermophysical Properties of Semiconductors (Atom Publ. House, Moscow, 1972), https://inis.iaea.org/search/search .aspx?orig_q=RN:5103404.

[33] C. J. Glassbrenner and G. A. Slack, Thermal Conductivity of Silicon and Germanium from $3^{\circ} \mathrm{K}$ to the Melting Point, Phys. Rev. 134, A1058 (1964).

[34] B. S. Hemingway, Quartz: Heat capacities from 340 to $1000 \mathrm{~K}$ and revised values for the thermodynamic properties, Am. Mineral. 72, 273 (1987), https://pubs.er.usgs.gov/ publication/70014245.

[35] Y. S. Touloukian, R. W. Powell, C. Y. Ho, and P. G. Klemens, Thermophysical Properties of Matter (IFI/Plenum, New York, 1970), Vol. 2, p. 193, https://apps.dtic.mil/sti/ citations/ADA951936.

[36] G. T. Furukawa, M. L. Reilly, and J. S. Gallagher, Critical analysis of heat-capacity data and evaluation of thermodynamic properties of Ruthenium, Rhodium, Palladium, Iridium, and Platinum from 0 to $300 \mathrm{~K}$. A survey of the literature data on osmium, J. Phys. Chem. Ref. Data 3, 163 (1974).

[37] D. Nečas, P. Klapetek, V. Neu, M. Havlíček, R. Puttock, O. Kazakova, X. Hu, and L. Zajíčková Determination of tip transfer function for quantitative MFM using frequency domain filtering and least squares method, Sci. Rep. 9, 3880 (2019).

[38] X. Hu, G. Dai, S. Sievers, V. Neu, and H. W. Schumacher, Uncertainty propagation and evaluation of nano-scale stray field in quantitative magnetic force microscopy measurements, 2018 Conference on Precision Electromagnetic Measurements (CPEM 2018), Paris (2018), pp. 1-2.

[39] P. J. Schendel, H. J. Hug, B. Stiefel, S. Martin, and H.-J. Güntherodt, A method for the calibration of magnetic force microscopy tips, J. Appl. Phys. 88, 435 (2000).

[40] W. Legrand, N. Ronceray, N. Reyren, D. Maccariello, V. Cros, and A. Fert, Modeling the Shape of Axisymmetric Skyrmions in Magnetic Multilayers, Phys. Rev. Applied 10, 064042 (2018).

[41] O. Kazakova, R. Puttock, C. Barton, H. Corte-León, M. Jaafar, V. Neu, and A. Asenjo Frontiers of magnetic force microscopy, J. Appl. Phys. 125, 060901 (2019).

[42] S. Zhang et al. Direct writing of room temperature and zero field skyrmion lattices by a scanning local magnetic field, Appl. Phys. Lett. 112, 132405 (2018).

[43] A. Casiraghi et al. Individual skyrmion manipulation by local magnetic field gradients, Commun. Phys. 2, 145 (2019).

[44] K. Manna, Y. Sun, L. Muechler, J. Kübler, and C. Felser, Heusler, Weyl and Berry, Nat. Rev. Mater. 3, 244 (2018).

[45] B. Binz and A. Vishwanath, Chirality induced anomalousHall effect in helical spin crystals, Physica (Amsterdam) 403B, 1336 (2008).

[46] N. Nagaosa and Y. Tokura, Topological properties and dynamics of magnetic skyrmions, Nat. Nanotechnol. 8, 899 (2013).

[47] H. Y. Nguyen, W. P. Pratt, and J. Bass, Spin-flipping in $\mathrm{Pt}$ and at Co/Pt interfaces, J. Magn. Magn. Mater. 361, 30 (2014).

[48] Z. Wang et al. Thermal generation, manipulation and thermoelectric detection of skyrmions, National electronics review 3, 672 (2020). 